Original Research Paper

\title{
Reverse Pharmacology-A Paradigm Shift for Drug Discovery and Development
}

\author{
Ashok D.B. Vaidya \\ Kasturba Health Society, Advanced Centre of Reverse Pharmacology (ICMR) 17, Khandubhai Desai Road, Vile Parle (West), \\ Mumbai, 400 056, India \\ Department of Pharmaceutical Sciences, Saurashtra University, India
}

Article history

Received: 06-11-2014

Revised: 28-11-2014

Accepted: 05-12-2014

Corresponding Author: Ashok D.B. Vaidya

Kasturba Health Society, Advanced Centre of Reverse Pharmacology (ICMR) 17, Khandubhai Desai Road, Vile Parle (West),

Mumbai, 400 056, India

Email: mrckhs@gmail.com

\begin{abstract}
The current scenario of drug discovery and development is facing a model perturbation, despite significant advances in genomics, proteomics, metabolomics, combinatorial chemistry and high through put assays. There is an urgent need of a paradigm shift in approaches to drug discovery. Natural products of Ayurveda offer a vast potential for novel phytomolecules with clinical activity. In India, Ayurveda is availed of by more than $70 \%$ of the population. There has been a renaissance in scientific exploration of medicinal plants with therapeutic activity. New methods have been proposed and developed for such exploration. Ayurvedic Pharmacoepidemiology, Observational therapeutics and Reverse Pharmacology paths have led to significant hits, leads and drug candidates for several diseases. The active phyto-molecules will also provide novel scaffolds for medicinal chemists to enhance efficacy and reduce toxicity. Several plants have shown promise Viz. Mucuna pruriens for Parkinson's disease, Nycthanthes arbor-tritis for malaria and Phyllanthus amarus for hepatitis. This approach of Reverse Pharmacology has also been adopted globally by several groups. A plea is made to look at the therapeutic activity of natural products for a revolution in drug discovery and development.
\end{abstract}

Keywords: Reverse Pharmacology, Ayurveda, Drug Discovery and Development

\section{Introduction}

A life-long pursuit in the domain of drug discovery and development, from the New Chemical Entities (NCE) and medicinal plants, obviously led to a serious introspection on the ruling paradigms and conventional approaches, followed globally, by the pharmaceutical industry. The experience of conducting phase 1,2 and 3 studies on more than a dozen drug candidates, discovered at CIBA-Geigy Research Centre at Mumbai, provided a ringside view of the failures and success of the game. Unlike the human studies with NCEs, the medicinal plants from Ayurveda had the benefit of their human safety and therapeutic activity in Ayurveda. This fact led to a fresh exploration of the history of modern medicine. It was realized that human observations on several natural products had ignited the curiosity of investigators. Understanding the mechanisms of the plant actions opened up the paths for the medicinal chemistry and screening of NCEs based on the phytomolecules. It was then felt that a paradigm shift was needed in the approaches in new drug discovery and development. We must and can refocus on the novel biodynamic effects of natural products in human as starting points for drug discovery.

Gilbert (1993) cited Sir Austin Bradford Hill, the pioneer of Medical Statistics and clinical drug trial, "All scientific work is incomplete-whether it be observational or experimental. All scientific work is liable to be upset or modified by advancing knowledge. That does not confer upon us a freedom to ignore the knowledge we already have or postpone the action that it appears to demand at a given time." It is in the emphasis on not 'to ignore the knowledge we already have that the path of Reverse Pharmacology (RP) finds a rationale as a new paradigm. Clinical anecdotes, robustly documented, constitute the knowledge we have and cannot be neglected. Clinical Pharmacology can become a discovery discipline and not a mere handmaid to the pharmaceutical as a conduit to new drug registrations. Bradford Hill listed the criteria of causality which can be applied to novel bedside pharmacodynamic and toxic effects to understand and pursue the phenomena at other 
levels of biological organization. The nine view-points proposed by Hill (1965) are: (1) Strength of association, (2) Temporal relationship, (3) Consistency, (4) Theoretical plausibility, (5) Coherence, (6) Specificity in the causes, (7) Dose response relationship, (8) Experimental evidence and (9) Analogy ${ }^{3}$. While studying the bedside drug-induced novel finding we can apply these criteria to Ayurvedic Pharmacology as suggested by Vaidya et al. (2003) However we must not forget he caution expressed by Doll (1992), "None of these nine viewpoints can bring indisputable evidence for or against a cause and effect hypothesis .... What they can do, with greater or less strength, is to help answer the fundamental question-is there any other way of explaining the set of facts before us, is there any other answer equally, or more, likely than cause and effect?" Vaidya (2011) suggested that observational therapeutics in Ayurveda can provide such identified hits for new drug discovery through RP.

\section{Direct Pharmacology Path for Drug Discovery}

For many years, the pharmaceutical new drug discovery was dominated by 'medicine-naïve chemists' to fulfil the expectations of 'chemistry-naïve doctors'. Now-a-days the ruling paradigm is based on genomics, proteomics and metabolomics. These new kids on the block are often both medicine-naive and chemistrynaive! No wonder the attrition rate of the new drug candidates is so high. The average costs of new drug discovery are stated to be around $\$ 1.3$ billion. The non-specific OECD guideline for toxicity studies, commercial clinical trials by contract research organizations and the lack of physician involvement in new drugs are some of the reasons for the present debacle in drug discovery. The developing nations are frightened away from the domain by the costly and long process of getting a new drug on the market. The $0.01 \%$ market yield from the baseline of $100 \%$ NCEs makes one wonder for the need of a paradigm shift in drug discovery process.

\section{A Paradigm Shift in Drug Discovery}

Kuhn (1962) wrote, "The historian of science may be tempted to exclaim that when paradigms change, the world itself changes with them." In our proposal of 'bedside to the bench', we expect that the clinical ward to evolve from a service-teaching unit to a clinical research milieu. The entrenched paradigm of normal science, over a period, fails to deliver results and discoveries as in the earlier times. Then a model drift sets in, which can lead to model crisis. The crisis precipitates a revolution in the model, leading to a paradigm change. The entire cycle is called the Kuhn cycle. As the model of drug discovery and development has drifted and a crisis is imminent, Reverse Pharmacology from the traditional medicine is emerging as a model revolution. This paradigm change may not appear too impressive but offers the path to tap the therapeutic wisdom of millennia for the discovery of new drugs. Rabindranath Tagore has rightly said, "What is huge is not great. And pride is not everlasting."

\section{The Wisdom of Ayurveda}

India, the world's largest democracy has a live and vibrant traditional system of medicine-Ayurveda. Around $70 \%$ of India's 1.25 billion population uses Ayurveda for their healthcare. There is a separate Department of AYUSH, under the Ministry of Health and Family Welfare of the Government of India. The department has the education, services and research in Ayurveda, Yoga, Unani, Siddha and Homeopathy. There are more than 400 undergraduate and 100 postgraduate colleges of these systems. The registered trained physicians if these systems are more than 7.5 million. The vast live experience in these systems offers opportunities for drug discoveries. Figure 1 shows the spectrum of remedies used in Ayurveda.

There are more than 9000 manufacturers of Ayurvedic drugs in India. The traditional knowledge digital library (CSIR) has a database of thousands of Ayurvedic formulations. Millions of Indians consume hundreds of plants or formulations as self-medication or as home culinary items. Despite the widespread use of Ayurvedic plants, in India, there is not any appropriate documentation of the responses and reactions to these drugs. The classical Ayurvedic texts and teachers explain the drug effects in terms of Ayurvedic Drvyagunasproperties of the materials- as per their epistemology. Reverse Pharmacology would investigate the drug actions in terms of the modern pharmacology.

The poet-saint Dave (1998) stated once, "There are two paths to discover botanical drugs. One is the ancient traditional path of understanding the Guna-dharmas and then carry out the experiments in bedside therapeutics. The second path lies in the modern laboratory approach. It is very essential to synergize both the paths, bereft of stark commercial interest with a reverence for the herbs as our partners in the healing art." Vaidya and Vaidya (2006) described that Lokmanya Tilak, the father of Indian independence movement, had said, "Ayurvidya is an active and open ended interface of Ayurveda with the advancing modern sciences, especially life sciences." Recently, in India, a significant renaissance is taking place by a multi-pronged research in Ayurveda. The diverse paths for natural products Research and Development (R and D) are shown in the Fig. 2. 
(Sources: Herbal, mineral and animal)

\section{Dietary substrates: Foods and pathyas}

\section{Dietary supplements: Simple herbals and ayurceuticals}

\section{Drugs: Complex herbals, herbo-minerals and bhasmas}

Fig. 1. Spectrum of ayurvedic remedies

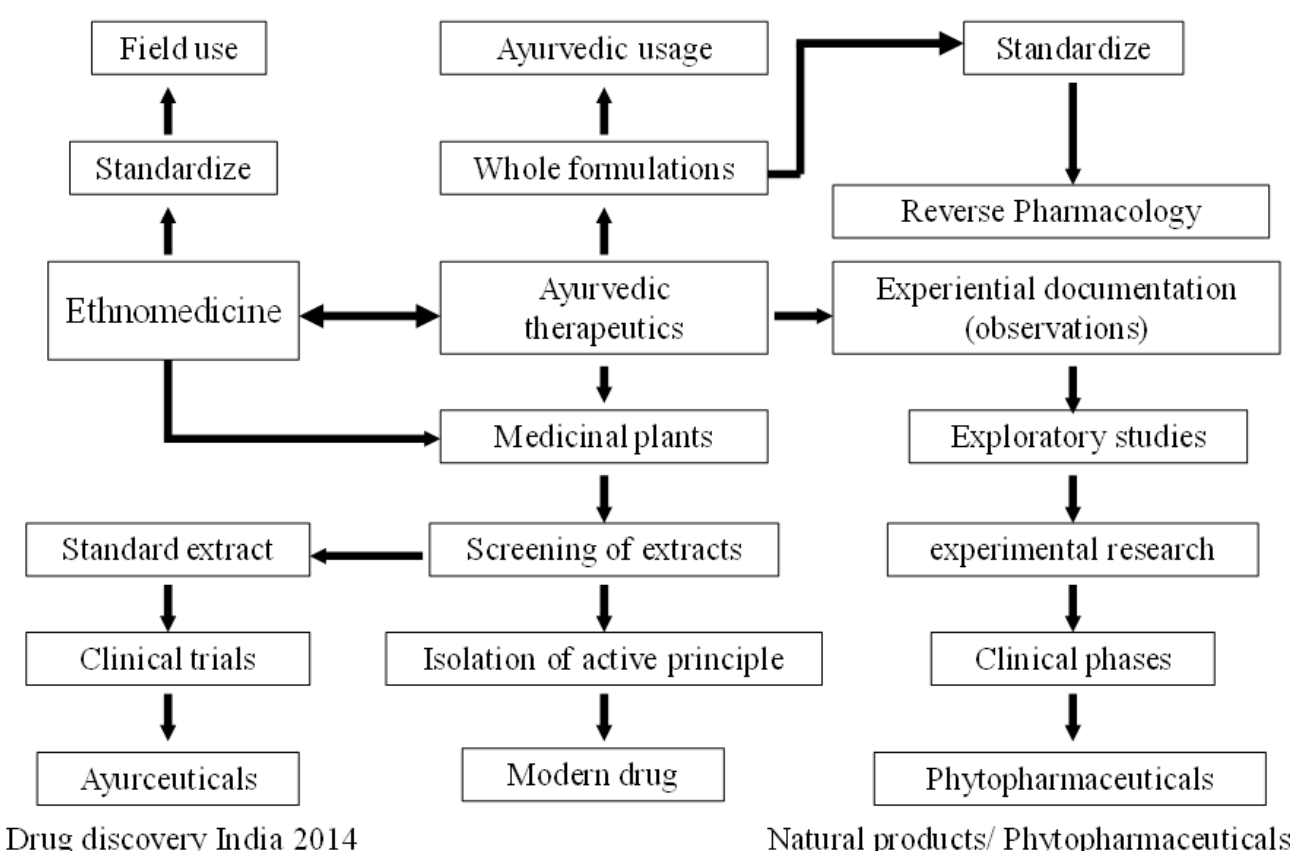

Fig. 2. $\mathrm{R}$ and $\mathrm{D}$ paths for natural products

\section{Reverse Pharmacology}

The definition of reverse pharmacology is as follows. Reverse Pharmacology (RP is the science of integrating bedside documented experiential hits into leads by trans-disciplinary exploratory studies (in vitro and in vivo) and to further develop these leads into drug candidates by state-of-the art experimental and clinical research. The scope of RP is to understand the mechanism of action at multiple levels of biological organization and to optimize safety, efficacy and acceptability of the leads from natural products, based on relevant science. The phytoactive compounds can serve as chemical scaffolds for novel medicinal chemistry. There are three major domains of RP; (1) the experiential domain covers literature search, Ayurvedic Pharmacoepidemiology and well-defined modest observational therapeutic studies with objective targets of response, (2) the exploratory animal studies cover indication-relevant in vivo and in vitro studies for efficacy and safety and human pharmacology and phase 2 studies for dose-finding, (3) the experimental domain involves well-planned experimental and clinical investigation at different levels of biological organization, extended clinical trials and sub-acute safety studies in two animal species. RP has emerged as a trans-disciplinary approach to pursue bedside observations back to the laboratory bench for new drugs. The approach can be cost-effective and fructify the leads from natural products. Reverse pharmacology can be a bridge for translational medicine from the traditional medicine to clinical practice. Such an effort would make healthcare delivery locally relevant and may lead to global discoveries in natural products. But it is essential that we develop multidisciplinary Ra nd D teamwork and infrastructure for reverse pharmacology. $\mathrm{T}$ he experts needed for RP are from Ayurveda, clinical pharmacology, clinical speciality, biomedical statistics, phytochemistry, pharmaceutics, 
experimental pharmacology, toxicology and molecular biology. Such a team is often hard to come by at any one institute. That was the reason that CSIR-NMITLI programme involved several institutes, industries and government laboratories in a nationwide $\mathrm{R}$ and $\mathrm{D}$ network. Table 1 shows the targets and indications taken up by CSIR-NMTLI. Several drug candidates have emerged with the programme.

\section{Reverse Pharmacology: Global Impact}

I was invited by M4 of NIH to make a presentation on the RP of anti-malarial Ayurvedic plants. Merlin Wilcox was also present in the audience. Willcox et al. (2007), later, worked on Argemone Mexicana as an anti-malarial phytomedicine by using a 'reverse pharmacology' approach Wilcox stated, "This example of 'reverse pharmacology' shows that a standardized phytomedicine can be developed faster and more cheaply than conventional drugs." The plant has been used in India and Africa in the traditional system of medicine and as folklore respectively. Benzylisoquinoline alkaloids-berberine, sangunarine and jatrorrhizine-are the active phytomolecules. Talwalkar et al. (2005) have used berberine to fluoresce the intra-erythrocytic malarial parasite.

Young-Joon (2011) from South Korea wrote an advocacy article on Reverse Pharmacology. Aggarwal et al. (2011) wrote an extensive review on novel antiinflammatory agents from Ayurveda, emphasizing the Reverse Pharmacology approach: He has listed 175 Ayurvedic plants and has provided 922 references. He stated, "We found that Ayurveda, a science of long life, almost 6000 years old, can serve as a "goldmine" for novel anti-inflammatory agents used for centuries to treat chronic diseases."

Patwardhan and Mashelkar (2009), while discussing the traditional medicine-inspired approaches to drug discovery from Ayurveda, elaborated on Reverse Pharmacology and Systems Biology ${ }^{15}$ Recently on July 7 to 14,2014 , a conference was organized at Chicago on natural products-A celebration and resurrection with Reverse Pharmacology, where Mukund Chorghade and Ashwin Raut stressed, "Standardized botanical products can be explored on a fast track basis using innovative approaches such as reverse pharmacology, systems biology and Information Technology."

The Maharashtra University of Health Sciences has initiated a postdoctoral fellowship training programme and diploma course in Reverse Pharmacology. This is the first such academic undertaking in the world. The Indian Council of Medical Research awarded the Advanced Centre of Reverse Pharmacology to our Kasturba Health Society. The centre has conducted RP studies in diabetes, arthritis, hepatitis, malaria and cancer. Several hits, leads and drug candidates have emerged for further research and development.

\section{Success Stories of Medicinal Plants}

There has been several success stories of drugs developed from medicinal plants prior to the proposed organized approach of RP. But the history makes it obvious how much opportunity loss occurred because of the time lag between the traditional use and the modern drug. The glaring examples are Artemisia, Cinchona and Ephedra etc. Sometimes the prevalent prejudice, cultural arrogance or ignorance prevented any attention to the systems alien to one's world-views. The other factor was the non-inclusion of the knowledge and traditions of other nations into the organized libraries and data bases.

Rauwolfia serpentina was demonstrated as an antihypertensive drug clinically as well as experimentally by Sen and Bose (1931) at Kolkatta. But the world did not take any notice of it until my teacher Vakil (1949) published a paper in the British Heart Journal. The clinical side effects of $R$ serpentina were meticulously reported by Sen Viz. depression, Parkinsonism and gynecomastia etc. As Kuhn has said, "Discovery commences with the awareness of anomaly, i.e., with the recognition that nature has somehow violated the paradigm-induced expectations that govern normal science.' The watershed in new drugs for depression, Parkinson's disease and galactorheea emerged several decades later from 'the anomalies' observed by Sen. Many more plants which show clinical effects need to be pursued systematically and in a time bound manner by RP.

There are interesting clinical effects of Ayurvedic drugs which have been studied in details. Table 2 lists some of the selected Ayurvedic plants and their relevant effects.

Table 1. Csir-nmitli Projects (2002-07)

\begin{tabular}{lll}
\hline Diabetes mellitus & Hepatitis & Arthritis \\
\hline Insulin resistance & Cytoprotective & Analgesic \\
Dyslipidaemia & Hydrocholeretic & Anti-inflammatory \\
Cataract & Anti-fibrotic & Chondro-protective \\
Reduced glycation & Immuno-modulator & Reduce TNF- $\alpha$ \\
Endothelial dysfunction & Steatosis & Sarcopenia \\
Angiogenesis & Antiviral & Disease modifying \\
\hline
\end{tabular}


Table 2. Reverse pharmacology of Ayurvedic plants

\begin{tabular}{|c|c|c|c|}
\hline Ayurvedic plants & Usage & Mechanism & Reference \\
\hline Withania somnifera & Strength giving & Muscle force & Raut et al. (2012) \\
\hline Nyctanthes arbour-tristis & Fever & Anti-plasmodial & Karnik et al. (2008) \\
\hline Tinospora cordifolia & Infections & Hepatoprotective & Rege et al.(1984) \\
\hline Mucuna pruriens & Fertility-enhancing & L-dopa & Vaidya et al. (1978) \\
\hline Phyllanthus amarus & Liver tonic & Anti-viral & Thyagarajan et al. (1998) \\
\hline
\end{tabular}

\section{Reverse Pharmacology of Drugs with New Indications}

Bedside observations on the established drugs may also have novel responses to consider. The path of RP can be applied effectively to the studies on the new indications for old drugs. The tragedy with thalidomideinduced phocomelia had alarmed the world and led to stringent regulatory requirements. But the discovery of a safe use of the optical isomer in multiple myeloma has been a great advance. Several such examples are known in the medical literature: Botulinum toxin for spasmodic torticolis, Hydroxyurea for the induction of fetal haemoglobin, aspirin for platelet aggregation inhibition and vitamin $\mathrm{K}_{2-7}$ for muscle cramps. The constant vigilance at the bedside may offer many more such opportunities for the enlargement of indications of known drugs. The stigma attached to use of known drugs for unauthorized indications can be overcome in a fast track by the application of RP to the problem.

\section{Future Scope and Conclusions}

The current paradigm of drug discovery is shaken by the failure rates of the drug candidates at different stages of development. The longstanding traditional wisdom of Ayurveda can be explored for drug discovery and development. The experiential data can be explored further by relevant in vivo and in vitro experiments. This trans-disciplinary approach of Reverse Pharmacology has sizeable scope for new natural drugs and their derivatives. Efforts in this direction are already showing promise. The trans-discipline of RP needs a regulatory recognition and support for its development. We have to relook at the novel human biodynamic effects and pursue these for their therapeutic potential. Asia-Africa can offer RP for a better integrated health-care.

\section{Author's Contributions}

All authors equally contributed in this work.

\section{Ethics}

This article is original and contains unpublished material. The corresponding author confirms that all of the other authors have read and approved the manuscript and no ethical issues involved.

\section{References}

Aggarwal, B., S. Prasad, S. Reuter, R. Kannappan and V. Yadev et al., 2011. Identification of novel antiinflammatory agents from Ayurvedic medicine for prevention of chronic diseases: "Reverse pharmacology" and "bedside to bench" approach. Curr. Drug Targets., 12: 1595-1653. PMID: 2156142

Dave, M., 1998. Personal Communication.

Doll, R., 1992. Sir Austin Bradford hill and the progress of medical science. Brit. Med. J., 305: 1521-1526. PMID: 1286370

Gilbert, S., 1993. Sir Austin Bradford Hill. Statis. Med. 12: 795-808.

Hill, B.A., 1965. The environment and disease: Association or causation? Proc. Roy. Soc. Med., 58: 295-300. PMID: 1898525

Karnik, S.R., P. Tathed, D. Antarkar, C. Godse and R. Vaidya et al., 2008. Antimalarial activity and clinical safety of traditionally used Nyctanthes arbor-tristis linn. Ind. J. Trad. Know., 7: 330-334.

Kuhn, T.S., 1962. The Structure of Scientific Revolutions. 1st Edn., University of Chicago Press, Chicago, USA, ISBN-10: 0226458040, pp: 172.

Patwardhan, B. and R. Mashelkar, 2009. Traditional medicine-inspired approaches to drug discovery: Can Ayurveda show the way forward? Drug Disc. Today, 14: 804-811. DOI: $10.1016 /$ j.drudis.2009.05.009

Raut, A.A., N. Rege, F. Tadvi, P. Solanki and K. Kene et al., 2012. Exploratory study to evaluate tolerability, safety and activity of Ashwagandha (Withania somnifera) in healthy volunteers. J. Ayur. Integ. Med., 3: 111-114. DOI: 10.4103/0975-9476

Rege, N.N., S. Dahanukar and S. Karandikar, 1984. Hepatoprotective effects of Tinospora cordifolia against carbon tetrachloride induced liver damage. Ind. Drugs, 21: 544-580.

Sen, G. and K. Bose, 1931. Rauwolfia serpentina, a new Indian drug for insanity and high blood pressure. Ind. Med. Wld., 2: 194.

Talwalkar, S., A. Vaidya, C. Godse, A. Vaidya and R. Vaidya. 2005. Plasmodium dna fluoresces with berberine a novel approach for diagnosis of malarial parasites. Am. J. Clin. Pathol., 124: 408-412. DOI: 10.1309/CG7037YYPBUBV703 
Thyagarajan, S.P., T. Thirunalasundari, S. Subramanian, P.S. Venkateswaran and B.S. Blumberg. 1998. Effect of Phyllanthus amarus on chronic carriers of hepatitis b virus. Lancet, 332: 764-766. DOI: 10.1016/S0140-6736(88)92416-6

Vaidya, A. and R. Vaidya, 2006. Pharmacology at a reverse. Express Pharma, 16-30.

Vaidya, A.B., T.G. Rajagopalan, N. Mankodi, D. Antarkar and P. Tathed et al. 1978. Treatment of parkinson's disease with the cowhage plantMucuna pruriens bak. Neurol. India, 26: 171-76. PMID: 753996

Vaidya, R.A., 2011. Observational therapeutics: Scope, challenges and organization. J. Ayurveda. Integr. Med., 2: 165-169. DOI: 10.4103/0975-9476.90764
Vaidya, R.A., A.D.B. Vaidya, B. Patwardhan, G. Tillu and Y. Rao, 2003. Ayurvedic pharmacoepidemiology: A proposed new discipline. J. Associat. Phy. India, 51: 528. PMID: 12974443

Vakil, R.J., 1949. A Clinical trial of Rauwolfia serpentina in essential hypertension. Br. Heart J., 11: 350-355. DOI: 10.1136/hrt.11.4.350.

Willcox, M.L., B. Graz, J. Falquet, O. Sidibe and M. Forster et al., 2007. Argemone mexicana decoction for the treatment of uncomplicated falciparum malaria. Trans. Roy. Soc. Trop. Med. Hyg., 101: 1190-1198. DOI: 10.1016/j.trstmh.2007.05.017

Young-Joon, S., 2011. Reverse pharmacology. Applicable for botanical drug developmentinspiration from the legacy of traditional wisdom. J. Tradit. Complement. Med., 1: 5-7. PMID: 13239630 